\title{
Construction of Lyapunov Functions Using Gröbner Bases
}

\author{
K. Forsman \\ Dept. of EE, Linköping University, S-581 83 Linköping, SWEDEN \\ Email: krister@isy.liu.se
}

\begin{abstract}
In this paper we show how so called Gröbner bases can be used when choosing parameters in Lyapunov functions for nonlinear dynamical systems in an optimal way. The method suggested requires the nonlinearities of the system and the Lyapunov function to be of polynomial type. Some concrete examples of how to apply the method is provided.
\end{abstract}

Keywords: Lyapunov theory, polynomial systems, constructive commutative algebra, optimization

\section{Introduction}

There exists a large literature on how to construct Lyapunov functions for nonlinear dynamical systems. Some classical methods such as Zubov's and Krasovski's methods are presented in [9]. For some more recent work, see e.g. $[3,4,8,10]$.

In this paper we give yet another contribution to this variety of methods, based on optimization. The present method uses so called Gröbner bases which can be said to be the analogue of Gaussian elimination to systems of polynomial equations, simply put.

We will assume that the reader has some familiarity with elementary concepts in commutative algebra, such as ideals.

\section{Gröbner Bases}

One of the most important tools in constructive commutative algebra today are so called Gröbner bases (GB). There is no room here to give a detailed description of Gröbner bases so for this we refer to [1]. Here we will use them merely for eliminating variables in systems of polynomial equations and hereby solve these equations.

The most important property to us is th $\quad$ GB for an ideal $I$ (with an appropriate choice of ranking) will tell us if there are elements in $I$ that are polynomials involving a subset of the ringvariables only, and even more: the degrees of the polynomials will also be minimal!

Gröbner bases are always finite, and there exist algorithms that compute the GB of any ideal, given a set of generators. These algorithms have been implemented on computers in several different versions, for instance in the symbolic algebra program Maple [2], which has been used here.

\section{Local Lyapunov Functions}

Consider a dynamical system

$$
\dot{x}=f(x), \quad x=\left(x_{1}, \ldots, x_{n}\right), \quad f: \mathbb{R}^{n} \rightarrow \mathbb{R}^{n}
$$

where each component of $f$ is polynomial $f_{i} \in k\left[x_{1}, \ldots, x_{n}\right]$. Suppose that we have a polynomial parametrized Lyapunov function $V(\eta, x)$ for this system

$$
V: \mathbb{R}^{n} \rightarrow \mathbb{R}, \quad V \in k\left[\eta, x_{1}, \ldots, x_{n}\right]
$$

where $V\left(\eta^{0}\right)$ is a local Lyapunov function for a nominal value $\eta^{0}$ of $\eta \in \mathbb{R}^{q}$. We introduce the notation $Q=V_{x} \cdot f$, where $V_{x}$ denotes the gradient of $V$ w.r.t. $x$.
The regions $\Omega(\eta)$ and $B_{d}(\eta)$ are defined by

$$
\begin{aligned}
& \Omega(\eta)=\left\{x \in \mathbb{R}^{n} \mid Q(x, \eta) \leq 0\right\} \\
& B_{d}(\eta)=\left\{x \in \mathbb{R}^{n} \mid V(x, \eta) \leq d\right\}
\end{aligned}
$$

with $Q$ as above. It is a classical result [9] that the stability of $(1)$ is guaranteed within $B_{d}$ if $B_{d}(\eta) \subseteq \Omega(\eta)$.

To find out for which $\eta$ this is satisfied we can examine the ideal

$$
\left(V-d, Q, \frac{\partial V}{\partial x_{1}}-\lambda \frac{\partial Q}{\partial x_{1}}, \ldots, \frac{\partial V}{\partial x_{n}}-\lambda \frac{\partial Q}{\partial x_{n}}\right)
$$

For a motivation of this see for instance $[7,5]$.

Since we are interested in choosing the parameters $\eta$ it is appropriate to compute a Gröbner base $\mathbf{G}$ for the ideal (3) w.r.t. a ranking

$$
\left\{\eta_{1}, \ldots, \eta_{q}\right\} \prec\left\{x_{1}, \ldots, x_{n}, \lambda\right\}
$$

G typically contains elements that are polynomials in $\eta$. In this way we get information about which $\eta$ are allowed, and then we can apply some criterion for optimality to choose among these. We will show how to do this more concretely in the two examples below. For more details, see $[5,6]$.

\section{Some Examples}

Example 4.1 Let

$$
\begin{gathered}
f=\left(-x_{1}+5 x_{1}^{2} x_{2},-x_{2}\right) \\
V=(a+1) x_{1}^{2}+2(a-1) x_{1} x_{2}+(a+1) x_{2}^{2}
\end{gathered}
$$

Then $V=a$ defines a family of ellipses with major axis along the direction $(-1,1)$ and minor axis along $(1,1)$. Furthermore, $2 \sqrt{a}$ is the length of the major axis and the minor axis has length 2 . It is not difficult to check that $a^{0}=1$ can be taken as nominal parameter value. We now ask for what values of $a$ that $B_{a} \subset \Omega(a)$. Knowing this we will be able to answer the question "which is the largest stability region that can be obtained using the family of Lyapunov functions defined by (6)?"

The ideal (3) is in this case A Gröbner base for the ideal (3) w.r.t. the ranking $\lambda \succ x_{2} \succ x_{1} \succ a$ contains the polynomial

$$
p=175 a^{5}-600 a^{4}-3210 a^{3}-2624 a^{2}+675 a
$$

which has three real roots: $0,0.204 \ldots$ and $6.56 \ldots$ Since $a^{0}=1$ a valid choice for $a$ is $a \in[0.204,6.56]$. In figure 1 we show the situation in the case $a=6.56$. As expected, this is the borderline case. Since we have that $\alpha<\beta \Rightarrow B_{\alpha}(\alpha) \subset B_{\beta}(\beta)$ we conclude 


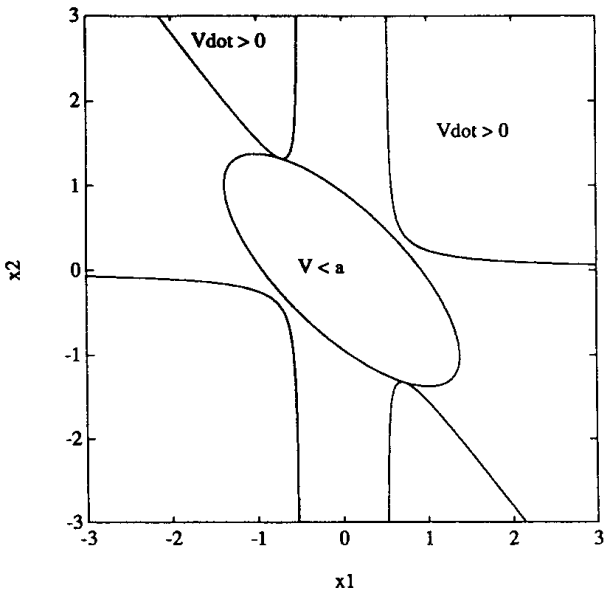

Figure 1: $B_{a}(a)$ and $\Omega(a)$ for $a=6.56$

that for the system (5) the optimal Lyapunov function in the family (6) is obtained by putting $a=6.56$.

Example 4.2 The system

$$
\dot{x}_{1}=-x_{1}+x_{2}^{2}, \quad \dot{x}_{2}=-x_{1}-2 x_{2}
$$

has a stable linearization around the origin. Again we study a one-parameter family of elliptic functions:

$$
V=x_{1}^{2}+q^{2} x_{2}^{2}
$$

Thus the parameter $q$ is the ratio between the lengths of the axes of the ellipse. Straightforward computations show that a $V$ in the family is a local Lyapunov function for (7) if $0<q<\sqrt{8}$. We now consider level curves of the form $V=q d$, so that the area of $B_{q d}$ is $\pi d$. The goal is to maximize $d$. The ideal

$$
I=\left(V-q d, Q, \frac{\partial V}{\partial x_{1}}-\lambda \frac{\partial Q}{\partial x_{1}}, \frac{\partial V}{\partial x_{2}}-\lambda \frac{\partial Q}{\partial x_{2}}\right)
$$

contains a large polynomial $p$ in $q$ and $d$ which we do not give explicitly here. We ask: of all points on the curve $p(d, q)=0$ which one has the maximum value of $d$ ? Thus we want a solution to the system

$$
p=0, \quad \frac{\partial p}{\partial q}=0
$$

Here the GB computations are too complex, so instead we have to use resultants. The resultant of the polynomials (10) is simply the discriminant of $p$ with respect to $q$, which can be computed fairly fast in Maple. Its positive zeroes are 16.14, 2.02, 89132. The largest one is not allowed, for it corresponds to $q=7.8$ which does not render a local Lyapunov function. However, for $d=$ 16.14 we get $q=1.74$ which is allowed.

But why not apply numerical methods directly to $(10)$ ? The answer is that the convergence is too messy: the sensitivity to the choice of initial values is very large, see [6].

In figure 2 we see part of the phase plane for the system together with the maximal $B_{d q}$. It can be seen that $\partial B_{d q}$ touches the separatrix at the stationary point $(4,-2)$.

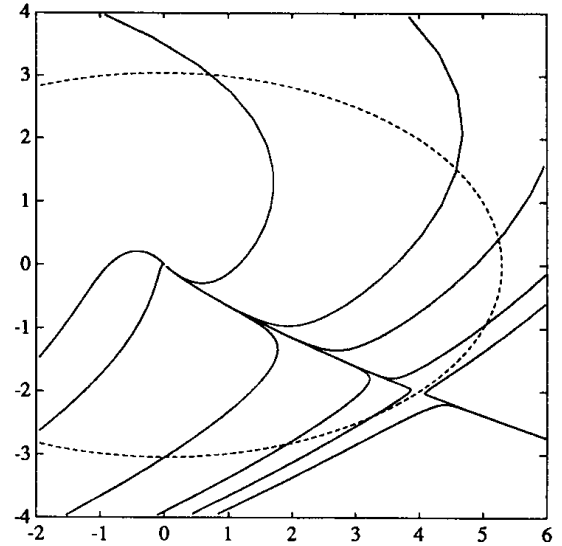

Figure 2: Phase plane for the system (7).

\section{References}

[1] B. Buchberger. Gröbner bases: An algorithmic method in polynomial ideal theory. In N.K. Bose, editor, Multidim. Systems Theory, pages 184-232. Dordrecht Reidel, 1985.

[2] B. Char, K.O. Geddes, G.H. Gonnet, M.B. Monagan, and S.M. Watt. Maple Reference Manual. Symbolic Computation Group, Univ. of Waterloo, fifth edition, March 1988.

[3] H.D. Chiang and J.S. Thorp. Stability regions of nonlinear dynamical systems: A constructive methodology. IEEE Trans. Aut. Contr., AC-34:1229-1241, December 1989.

[4] P.S.M. Chin. A general method to derive Lyapunov functions for non-linear systems. Int. J. Contr., 44(2):381-393.

[5] K. Forsman. Applications of Gröbner bases to nonlinear systems. In Proc. First European Control Conf., volume 1, pages 164-169, Grenoble, France, July 1991. Hermès.

[6] K. Forsman. Constructive Commutative Algebra in Nonlinear Control Theory. PhD thesis, Dept. Elec. Eng., Linköping Univ., S-581 83 Linköping, Sweden, 1991.

[7] K. Forsman and T. Glad. Constructive algebraic geometry in nonlinear control. In Proc. 29th $C D C$, volume 5, pages 2825-2827, Honolulu, Hawaii, 1990. IEEE CSS.

[8] R. Genesio, M. Tartaglia, and A. Vicino. On the estimation of asymptotic stability regions: State of the art and new proposals. IEEE Trans. Aut. Contr., AC-30(8):747755, August 1985.

[9] W. Hahn. Stability of Motion. Springer, 1967.

[10] D.N. Shields and C. Storey. The behaviour of optimal Lyapunov functions. Int. J. Contr., 21(4):561-573, 1975.

\section{Acknowledgement}

This work was supported by the Swedish Board for Technical Development (STU). 